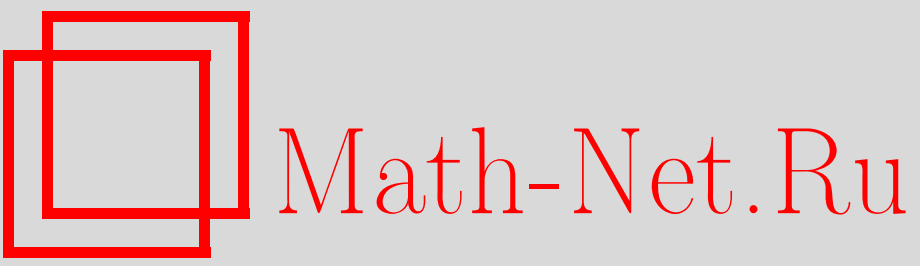

Е. В. Белокурова, Характеристические классы проективных модулей над комплексными полупростыми алгебрами, УМН, 1999, том 54, выпуск 4, 153-154

DOI: https://doi.org/10.4213/rm182

Использование Общероссийского математического портала Math-Net.Ru подразумевает, что вы прочитали и согласны с пользовательским соглашением

http://www.mathnet.ru/rus/agreement

Параметры загрузки:

IP: 54.162.27.143

26 апреля 2023 г., 14:04:51 


\title{
ХАРАКТЕРИСТИЧЕСКИЕ КЛАССЫ ПРОЕКТИВНЫХ МОДУЛЕЙ НАД КОМПЛЕКСНЫМИ ПОЛУПРОСТЫМИ АЛГЕБРАМИ
}

\author{
Е. В. БЕЛОКУРовА
}

В работе показано, что характеристические классы конечнопорожденных проективных модулей над комплексными полупростыми алгебрами равны нулю.

Рассмотрим комплексную ассоциативную алгебру $A$ с единицей. Пусть $Z$ - подалгебра, лежащая в центре алгебры $A, D$ - подалгебра алгебры Ли $\operatorname{Der}(A)$, замкнутая относительно действия $Z, V$ - некоторый $Z$-модуль, на котором действует алгебра $D$ и $\tau: A \rightarrow V$-отображение следа, являющееся гомоморфизмом $Z$-модулей и $D$-модулей и удовлетворяющее соотношению $\tau(a b)=\tau(b a)$.

Группы когомологий $H_{Z}^{*}(D, V)$ определим как когомологии комплекса $\Omega(D, V)$ :

$$
V \stackrel{\delta_{0}}{\longrightarrow} \operatorname{Hom}_{Z}\left(\Lambda_{Z}^{1}(D), V\right) \stackrel{\delta_{1}}{\longrightarrow} \operatorname{Hom}_{Z}\left(\Lambda_{Z}^{2}(D), V\right) \stackrel{\delta_{2}}{\longrightarrow} \cdots
$$

где дифференциал $\delta_{n}$ определяется стандартным образом.

Возьмем конечнопорожденный проективный правый $A$-модуль $E$. Связностью на модуле $E$ называется такое $\mathbb{C}$-линейное отображение $\nabla: E \rightarrow \operatorname{Hom}_{Z}(D, E)$, что $\nabla_{\partial}(\xi a)=\nabla_{\partial}(\xi) a+$ $\xi \partial(a)$, где $\xi \in E, \partial \in D, a \in A$ и $\nabla_{\partial}(\xi)=\nabla(\xi)(\partial)$. Форма кривизны связности $\Theta\left(\partial_{1}, \partial_{2}\right)=$ $\left[\nabla_{\partial_{1}}, \nabla_{\partial_{2}}\right]-\nabla_{\left[\partial_{1}, \partial_{2}\right]} \in \operatorname{End}_{A}(E)$.

Грассманова связность $\nabla^{0}$ имеет вид $\nabla_{\partial}^{0}(\xi)=P \partial(\xi), \xi \in E, P$ - проектор такой, что $\operatorname{Im} P=E$.

Справедлива формула [1]:

$$
\Theta^{0}\left(\partial_{1}, \partial_{2}\right) \xi=P\left(\partial_{1} P\right)\left(\partial_{2} P\right) \xi-P\left(\partial_{2} P\right)\left(\partial_{1} P\right) \xi
$$

Отображение следа $\tau: A \rightarrow V$ индуцирует гомоморфизм коцепных комплексов $\tau: \Omega(D, A) \rightarrow$ $\Omega(D, V)$ и продолжается на кольцо $\operatorname{End}_{A}(E)$.

Положим

$$
\sigma_{k}(\nabla, E)=\tau(\underbrace{\Theta \wedge \cdots \wedge \Theta}_{k \text { раз }}) .
$$

Справедливы утверждения [1], [2].

1) $\sigma_{k}(\nabla, E)$ является коциклом степени $2 k$ в комплексе $\Omega(D, V)$.

2) Классы когомологий $\operatorname{ch}_{k}(E)$ форм $\sigma_{k}(\nabla, E)$ не зависят от выбора связности на модуле $E$. Они называются характеристическими классами Чженя модуля $E$.

Теорема. Характеристические классы конечнопорожденных проективных модулей над комплексными полупростыми алгебрами равны нулю.

Для доказательства теоремы рассмотрим алгебру $A_{0}=M_{n}(\mathbb{C})$. Возьмем базис алгебры $A_{0}$, состоящий из единичной матрицы и $\left(n^{2}-1\right)$ эрмитовой матрицы $E_{k}$ с нулевым следом. Базис алгебры Ли $D=\operatorname{Der}\left(A_{0}\right)$ состоит из $n^{2}-1$ элемента $\partial_{k}$ :

$$
\partial_{k} E_{l}=\operatorname{ad}\left(i E_{k}\right) E_{l}=i\left[E_{k}, E_{l}\right]=2 C_{k l}^{m} E_{m} .
$$

Дуальный базис 1-форм состоит из форм

$$
\theta^{k}: D \rightarrow A_{0}, \quad k=1, \ldots, n^{2}-1 ; \quad \theta^{k}\left(\partial_{l}\right)=\delta_{l}^{k} I .
$$

Центр алгебры $A_{0} Z \simeq \mathbb{C}$. Пусть $V=\mathbb{C}, \partial v=0$ для любых $\partial \in D, v \in V$. Положим $\tau(a)=$ $\frac{1}{n} \operatorname{tr} a$.

Так как алгебра $A_{0}$ полупроста, то любой конечнопорожденный проективный $A_{0}$-модуль является прямой суммой неприводимых $A_{0}$-модулей. Единственным с точностью до изоморфизма 


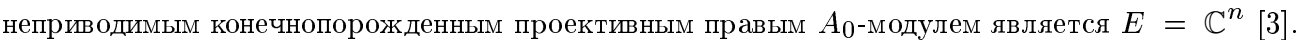
Пусть $E$ - это подмножество $A_{0}$, состоящее из матриц, у которых ненулевая только верхняя строка. Тогда $P=e_{1,1}-$ матричная единица,

$$
\begin{gathered}
\Theta^{0}=-4 i P\left(\theta^{n} \wedge \theta^{n+1}+\theta^{n+2} \wedge \theta^{n+3}+\cdots+\theta^{3 n-4} \wedge \theta^{3 n-3}\right), \\
\sigma_{1}\left(\nabla^{0}, E\right)=\tau\left(\Theta^{0}\right)=-\frac{4 i}{n}\left(\theta^{n} \wedge \theta^{n+1}+\theta^{n+2} \wedge \theta^{n+3}+\cdots+\theta^{3 n-4} \wedge \theta^{3 n-3}\right) .
\end{gathered}
$$

Заметим, что $\sigma_{1}\left(\nabla^{0}, E\right)=\delta\left(\frac{2 i}{n}\left(\theta^{1}+\cdots+\theta^{n-1}\right)\right)$. Поэтому класс когомологий $\operatorname{ch}_{1}(E)$ равен нулю. Ясно, что все $\operatorname{ch}_{k}(E)=0$ при $k \geqslant 1$. Таким образом, характеристические классы модулей над алгеброй $M_{n}(\mathbb{C})$ равны нулю.

Любая конечномерная комплексная полупростая алгебра $A$ изоморфна прямой сумме $M_{n_{1}}(\mathbb{C}) \oplus \cdots \oplus M_{n_{r}}(\mathbb{C})[4]$, при этом $\operatorname{Der}(A)=\operatorname{Der}\left(M_{n_{1}}(\mathbb{C})\right) \oplus \cdots \oplus \operatorname{Der}\left(M_{n_{r}}(\mathbb{C})\right)$. Составим базисы алгебр $A$ и $\operatorname{Der}(A)$ из описанных выше базисов $M_{n_{i}}(\mathbb{C})$ и $\operatorname{Der}\left(M_{n_{i}}(\mathbb{C})\right)$. Центр алгебры $A Z \simeq \mathbb{C}^{r}$. Возьмем $V=\mathbb{C}^{r}, \partial v=0$ для любых $\partial \in \operatorname{Der}(A), v \in V$. Положим $\tau(a)=\left(\frac{1}{n_{1}} \operatorname{tr} a_{1}, \ldots, \frac{1}{n_{r}} \operatorname{tr} a_{r}\right)$ для $a=\left(a_{1}, \ldots, a_{r}\right) \in A$.

$\mathrm{C}$ точностью до изоморфизма существуют $r$ неприводимых конечнопорожденных правых $A$-модулей [3] ( $i$-й модуль состоит из элементов алгебры $A$, у которых на $i$-м месте стоит матрица с ненулевой только верхней строкой, а на осталњных местах стоят нули). Повторяя для каждого модуля те же вычисления, что и для неприводимого $M_{n}(\mathbb{C})$-модуля, получаем доказательство теоремы.

Выражаю глубокую благодарность Ю.П. Соловьеву за постановку задачи и полезные консультации.

\section{СПИСОК ЛИТЕРАТУРЫ}

[1] Ж Кураев Ю. $\breve{И . ~ Д и с с . ~ . . . ~ к а н д . ~ ф и з .-м а т е м . ~ н а у к . ~ М .: ~ М Г У, ~ 1988 . ~[2] ~ Ж ~ у р а е в ~ Ю . ~} \breve{И ., ~}$ Мишенко А.С., Соловьев Ю.П. // Вестн. Моск. ун-та. Сер. 1. 1986. № 1. С. 75-76. [3] Шафаревич И. Р. Итоги науки и техн. Совр. пробл. матем. Т. 11. М.: ВИНИТИ, 1986. [4] Пирс Р. Ассоциативные алгебры. М.: Мир, 1986.

Московский государственный

Принято редколлегией университет им. М. В. Ломоносова

21.07.1999 